\title{
Icotinib Hydrochloride
}

National Cancer Institute

\section{Source}

National Cancer Institute. Icotinib Hydrochloride. NCI Thesaurus. Code C99160.

The hydrochloride salt form of icotinib, an orally available quinazoline-based inhibitor of epidermal growth factor receptor (EGFR), with potential antineoplastic activity. Icotinib selectively inhibits the wild-type and several mutated forms of EGFR tyrosine kinase. This may lead to an inhibition of EGFR-mediated signal transduction and may inhibit cancer cell proliferation. EGFR, a receptor tyrosine kinase, has been upregulated in a variety of cancer cell types. 